\title{
Development of load specifications for the design of the breeding blanket system
}

G. A. Spagnuolo ${ }^{1}$, L.V. Boccaccini ${ }^{1}$, G. Bongiovì ${ }^{1}$, F. Cismondi ${ }^{2}$, I. A. Maione ${ }^{1}$

${ }^{1}$ Karlsruhe Institute of Technology (KIT), Institute for Neutron Physics and Reactor Technology (INR)

${ }^{2} E U R O f u s i o n$ Consortium, Programme Management Unit

\section{Motivation}

L List of all relevant single loads and load combinations

Categorization of relevant load combinations

$\square$ Identification of the load combinations short list relevant to the Pre-Conceptual Design (PCD) phase

\section{- Load categories and damage levels}

\begin{tabular}{ccc}
\hline $\begin{array}{c}\text { Loading } \\
\text { Category }\end{array}$ & $\begin{array}{c}\text { Damage } \\
\text { Limit }\end{array}$ & $\begin{array}{c}\text { Criteria } \\
\text { Level }\end{array}$ \\
\hline $\begin{array}{c}\text { Category I: } \\
\text { Operational Loading } \\
\text { Conditions }\end{array}$ & $\begin{array}{c}\text { Normal } \\
\text { test }\end{array}$ & Level A \\
$\begin{array}{c}\text { Category II: Likely } \\
\text { Loading Conditions } \\
\left(f>10^{-2} / a\right)\end{array}$ & Upset & Level B \\
& &
\end{tabular}

Category III: Unlikely

Loading Conditions Emergency Level C $\left(10^{-2} / a>f>10^{-4} / a\right)$

Category IV:

Extremely Unlikely

Loading Conditions

$\left(10^{-4} / a>f>10^{-6} / a\right)$

$\square$ Criteria levels defined according to RCC-MRx

Events, with lower occurrence frequency than the Cat- IV, are not considered within the design basis

Short list of load combinations for the HCPB and WCLL BB for the PCD phase

\begin{tabular}{|c|c|c|c|c|c|}
\hline Cat. & $\begin{array}{c}\text { DEMO } \\
\text { Operat. } \\
\text { State }\end{array}$ & $\begin{array}{c}\text { Plasma } \\
\text { state }\end{array}$ & $\begin{array}{c}\text { Press.I } \\
\text { Magn.I } \\
\text { Seis. }\end{array}$ & $\begin{array}{l}\text { Initiat. } \\
\text { event }\end{array}$ & $\begin{array}{c}\text { Concaten } \\
\text { event }\end{array}$ \\
\hline I & POS & Normal cycle & - & - & - \\
\hline I & POS & $\begin{array}{l}\text { Fus. Power } \\
\text { excursion }\end{array}$ & - & - & - \\
\hline II & POS & MD II & - & MD II & - \\
\hline II & POS & VDE II & - & VDE II & - \\
\hline II & POS & MD I & SL-1 & SL-1 & MD I \\
\hline III & POS & MD III & - & MD III & - \\
\hline III & POS & VDE III & - & VDE III & - \\
\hline IV & POS & Normal cycle & SL-2 & SL-2 & - \\
\hline IV & POS & Normal cycle & - & - & $\begin{array}{c}\text { Ex-Vessel } \\
\text { LOCA }\end{array}$ \\
\hline IV & POS & Normal cycle & - & $\begin{array}{l}\text { In-Box } \\
\text { LOCA }\end{array}$ & - \\
\hline
\end{tabular}

\section{Path of the main loads}

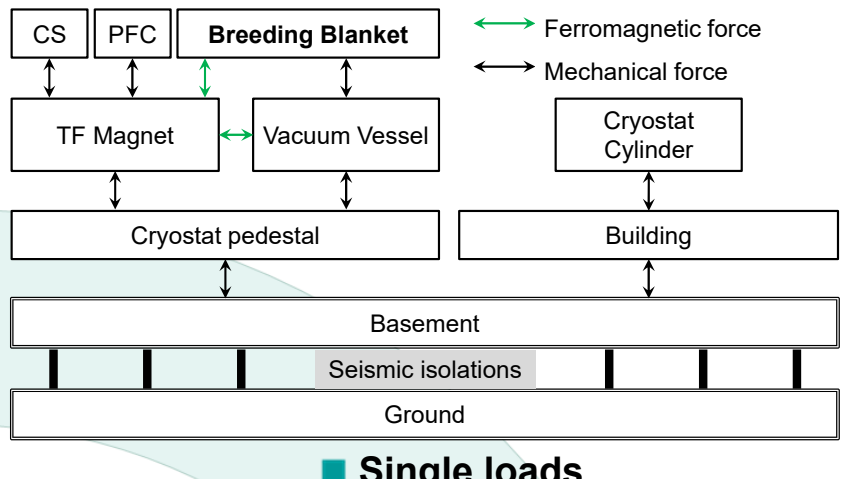

Breeding
Blanket

Load

Specifications

Document
Single loads

\begin{tabular}{cc}
\hline Load & Type \\
\hline Dead Weight & Inertial loads \\
Seismic & \\
State & \\
Test & \\
In-Vessel LOCA & Pressure loads \\
In-Box LOCA & \\
Ex-Vessel LOCA & \\
Normal operational &
\end{tabular}

Accidental events Thermal loads Off normal transients

Plasma pulse Off-normal

EM loads

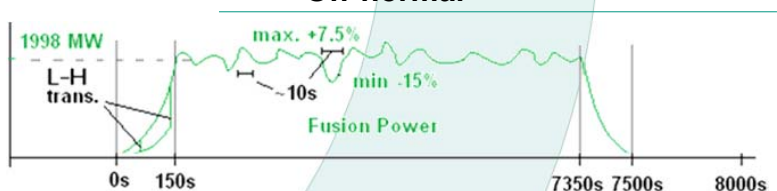

Pressure and temperature loads in each state

\begin{tabular}{|c|c|c|c|c|c|}
\hline \multicolumn{2}{|c|}{ Component } & Fluid & $\begin{array}{c}\text { Pressure } \\
\text { test }\end{array}$ & $\begin{array}{c}\text { Normal } \\
\text { Operation }\end{array}$ & $\begin{array}{c}\text { Design } \\
\text { value }\end{array}$ \\
\hline \multirow{4}{*}{$\begin{array}{c}\text { HCPB } \\
\text { BB }\end{array}$} & & & 17.80 & & \\
\hline & $\begin{array}{c}+ \\
\text { BZ }\end{array}$ & Helium & $20^{\circ} \mathrm{C}$ & & $550^{\circ} \mathrm{C}$ \\
\hline & & & $0.445 \mathrm{MPa}$ & $0.2 \mathrm{MPa}$ & $0.23 \mathrm{MPa}$ \\
\hline & & & & & \\
\hline \multirow{4}{*}{$\begin{array}{c}\text { WCLL } \\
\text { BB }\end{array}$} & FW & Water & $\begin{array}{r}25.57 \\
20\end{array}$ & $\begin{array}{l}\mathrm{MPa} \\
{ }^{\circ} \mathrm{C}\end{array}$ & $\begin{array}{r}17.82 \\
344\end{array}$ \\
\hline & \multirow{3}{*}{$B Z$} & & 25.57 & $\mathrm{MPa}$ & 17.8 \\
\hline & & & $20^{\circ} \mathrm{C}$ & $295^{\circ} \mathrm{C}$ & $344.8^{\circ} \mathrm{C}$ \\
\hline & & PbLi & $\begin{array}{c}0.742 \mathrm{MPa} \\
20^{\circ} \mathrm{C}\end{array}$ & $\begin{array}{c}0.45 \mathrm{MPa} \\
326^{\circ} \mathrm{C}\end{array}$ & $\begin{array}{c}0.5175 \mathrm{MPa} \\
344.8^{\circ} \mathrm{C}\end{array}$ \\
\hline
\end{tabular}

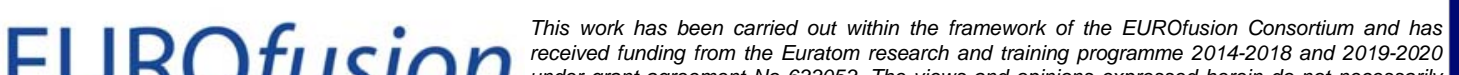

